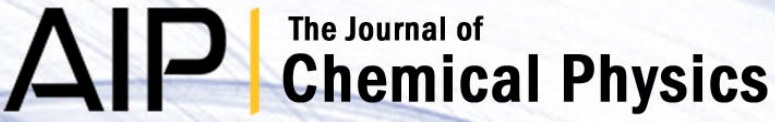

A reduced-dimensionality quantum model which incorporates the fulldimensional energy of the system: Application to the vibrational predissociation of $\mathrm{Cl} 2-\mathrm{Ne} 2$

M. Ceotto and A. García-Vela

Citation: J. Chem. Phys. 115, 2146 (2001); doi: 10.1063/1.1385153

View online: http://dx.doi.org/10.1063/1.1385153

View Table of Contents: http://jcp.aip.org/resource/1/JCPSA6/v115/i5

Published by the AIP Publishing LLC.

Additional information on J. Chem. Phys.

Journal Homepage: http://jcp.aip.org/

Journal Information: http://jcp.aip.org/about/about_the_journal

Top downloads: http://jcp.aip.org/features/most_downloaded

Information for Authors: http://jcp.aip.org/authors

\section{ADVERTISEMENT}

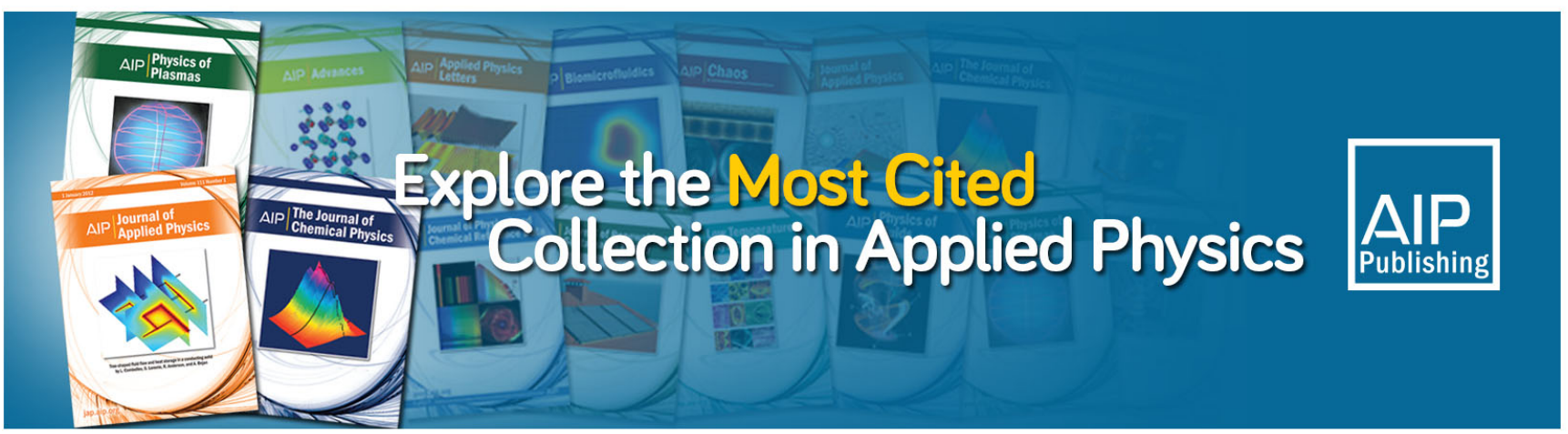




\title{
A reduced-dimensionality quantum model which incorporates the full-dimensional energy of the system: Application to the vibrational predissociation of $\mathrm{Cl}_{2}-\mathrm{Ne}_{2}$
}

\author{
M. Ceotto and A. García-Vela ${ }^{a}$ \\ Instituto de Matemáticas y Física Fundamental, C.S.I.C., Serrano 123, 28006 Madrid, Spain
}

(Received 28 March 2001; accepted 21 May 2001)

\begin{abstract}
A reduced-dimensionality quantum model is proposed which incorporates the zero-point energy of the neglected modes in a systematic, natural way. In this model the reduced-dimensionality Hamiltonian is obtained by averaging the exact Hamiltonian over the dependence of the full-dimensional initial state of the neglected modes. The reduced Hamiltonian conserves all the terms of the full Hamiltonian, providing a more flexible description of the couplings between the modes considered explicitly in the model. The model is applied to simulate the vibrational predissociation dynamics of $\mathrm{Cl}_{2}-\mathrm{Ne}_{2}$, considering the three stretching modes of the complex. The results are compared to experimental data and to previous calculations using a reduced-dimensionality quantum model and a full-dimensional quantum-classical approach. The $\mathrm{Cl}_{2}-\mathrm{Ne}_{2}$ resonance lifetimes obtained agree only qualitatively with the experimental and previously calculated ones. By contrast, the present model predicts more correctly than previous calculations the behavior of the $\mathrm{Cl}_{2}$ fragment vibrational distributions observed experimentally. The applicability of the model is discussed and further refinements are suggested. (C) 2001 American Institute of Physics. [DOI: 10.1063/1.1385153]
\end{abstract}

\section{INTRODUCTION}

In the last two decades a great deal of research effort has been devoted to investigate the fragmentation dynamics of van der Waals (vdW) clusters $\mathrm{BC}-\mathrm{Rg}_{n}(\mathrm{BC}=$ diatomic molecule, $\mathrm{Rg}=$ rare-gas atom). These systems are ideal candidates to study energy transfer processes which take place after energy is deposited in the diatomic subunit by optical excitation. Among such processes are intramolecular vibrational redistribution (IVR), vibrational predissociation (VP), and evaporative cooling. The chemical nature and the number of the rare gas atoms surrounding the diatomic chromophore influence the dynamics of energy transfer. In this sense, increasing gradually the number of rare gas atoms allows one to approach the condensed matter regime, and to explore solvation effects.

A variety of vdW clusters has been investigated experimentally using frequency-domain techniques. In the pioneering experiments of Levy and co-workers the structure and energy transfer mechanisms were investigated for $\mathrm{I}_{2}-\mathrm{He}_{n}$ $(n=1-3)^{1}$ and $\mathrm{I}_{2}-\mathrm{Ne}_{n}(n=1-7),{ }^{2}$ by measuring binding energies, vibrational predissociation linewidths and lifetimes, and spectral band shifts. The predissociation dynamics of other complexes like $\mathrm{Br}_{2}-\mathrm{Ne}_{n}(n=1-3),{ }^{3} \mathrm{ICl}_{-} \mathrm{Ne}_{n}(n$ $=1-5),{ }^{4} \mathrm{Cl}_{2}-\mathrm{Ne}_{n}(n=1-3),{ }^{5} \mathrm{Cl}_{2}-\mathrm{Ar}_{n}(n=1-3),{ }^{6}$ and $\mathrm{Cl}_{2}-\mathrm{He}_{n}(n=1,2),{ }^{7}$ was also studied. In the time domain, the VP of $\mathrm{I}_{2}-\mathrm{Ne}_{n}(n=1-4)$ complexes was investigated by Zewail and co-workers ${ }^{8}$ using real-time techniques.

The rich body of experimental information available poses on theory the challenge to develop models and methods to realistically simulate the energy transfer dynamics in $\mathrm{BC}-\mathrm{Rg}_{n} \mathrm{vdW}$ complexes. Classical and quasiclassical simu-

${ }^{a)}$ Electronic mail: garciavela@imaff.cfmac.csic.es lations of the VP dynamics of moderately large clusters like $\mathrm{I}_{2}-\mathrm{He}_{n}(n=1-9),{ }^{9} \mathrm{I}_{2}-\mathrm{Ne}_{n}(n=1-9),{ }^{10,11}$ and $\mathrm{I}_{2}-\mathrm{Ar}_{13},{ }^{12}$ have been carried out. Hybrid classical-quantum and quantum-classical methods have been applied to study the predissociation dynamics of $\mathrm{Cl}_{2}-\mathrm{He}_{2},{ }^{13} \mathrm{I}_{2}-\mathrm{Ne}_{n}$ $(n=2-6),{ }^{14}$ and $\mathrm{Cl}_{2}-\mathrm{Ne}_{n} \quad(n=2,3) .{ }^{15}$ Exact fulldimensional quantum calculations have been restricted to triatomic BC-Rg complexes. ${ }^{16-20}$ Quantum dynamical simulations on tetraatomic $\mathrm{BC}-\mathrm{Rg}_{2}$ clusters were performed using reduced-dimensionality models including three $\mathrm{e}^{21-23}$ and four $^{24,25}$ degrees of freedom. Exact quantum treatments of $\mathrm{BC}-\mathrm{Rg}_{n}$ systems including the full dimensionality are computationally out of reach for $n \geqslant 2$. Thus, in order to describe quantum effects in tetraatomic or larger vdW complexes, reliable reduced-dimensionality quantum (RDQ) models have to be developed.

In this paper we propose a RDQ model to simulate the VP dynamics of $\mathrm{BC}-\mathrm{Rg}_{2}$ systems. The model is applied to the $\mathrm{Cl}_{2}-\mathrm{Ne}_{2}$ complex. The predissociation dynamics of $\mathrm{Cl}_{2}-\mathrm{Ne}_{2}$ has been investigated experimentally, ${ }^{5}$ and theoretically by applying a time-dependent RDQ treatment ${ }^{22}$ and a hybrid quantum-classical method. ${ }^{15}$ These works provide a basis for comparison in order to test the results of our model. In previous RDQ approaches the zero-point energy of the neglected modes was either not included ${ }^{21,22}$ or included in an ad hoc way. ${ }^{23-25}$ This leads to a total energy of the complex which is different from the correct full-dimensional one. The model proposed here is close to that applied in Ref. 22, since the stretching vibrations of the complex are considered, while the bending modes are neglected.

The main novelty of our model is that it introduces in a systematic way the zero-point energy associated with the modes neglected. As a result, the reduced-dimensionality dynamical calculation is carried out at the total energy of the 
full-dimensional system. The total energy at which the reduced-dimensionality calculation is performed has an effect on the simulated energy transfer dynamics. Indeed, the Hamiltonian couplings responsible of the energy flow depend on the energy region accessed by the complex.

The paper is organized as follows. In Sec. II the VP process and the system are described, and the RDQ model is presented. Results on VP lifetimes of $\mathrm{Cl}_{2}-\mathrm{Ne}_{2}$ and vibrational distributions of the $\mathrm{Cl}_{2}$ fragment are shown and discussed in Sec. III. Conclusions are given in Sec. IV.

\section{THE FORMALISM}

\section{A. The VP process and the system}

The VP process in the $\mathrm{Cl}_{2}-\mathrm{Ne}_{2}$ complex occurs upon optical excitation of the $\mathrm{Cl}_{2}$ chromophore from the ground electronic state to a vibrational state $v>0$ in the excited $B$ electronic state. A resonance state $\mathrm{Cl}_{2}^{*}(B, v)-\mathrm{Ne}_{2}$ is prepared in this way, which decays to a dissociation continuum, leading to fragmentation of the complex. Fragmentation is caused by energy transfer from the $\mathrm{Cl}_{2}$ stretching vibration to the vdW modes. The fragmentation mechanism can follow different pathways:

$$
\begin{aligned}
& \mathrm{Cl}_{2}^{*}(B, v)-\mathrm{Ne}_{2} \rightarrow \mathrm{Ne}+\mathrm{Cl}_{2}\left(B, v^{\prime}<v\right)-\mathrm{Ne} \\
& \rightarrow \mathrm{Ne}+\mathrm{Ne}+\mathrm{Cl}_{2}\left(B, v^{\prime \prime}<v^{\prime}\right), \\
& \mathrm{Cl}_{2}^{*}(B, v)-\mathrm{Ne}_{2} \rightarrow \mathrm{Ne}+\mathrm{Ne}+\mathrm{Cl}_{2}\left(B, v^{\prime}<v\right), \\
& \mathrm{Cl}_{2}^{*}(B, v)-\mathrm{Ne}_{2} \rightarrow \mathrm{Ne}_{2}+\mathrm{Cl}_{2}\left(B, v^{\prime}<v\right) .
\end{aligned}
$$

The above pathways will be termed as sequential dissociation [Eq. (1)], simultaneous double dissociation [Eq. (2)], and production of two dimers [Eq. (3)]. The pathways of Eqs. (1)-(3) involve direct predissociation of the complex. Additional fragmentation pathways may occur where the energy initially transferred by the chlorine heats the vdW modes without breaking the complex. Further energy transfer produces either sequential or simultaneous dissociation of the two weak bonds in a rather evaporative way.

To a good approximation, the VP process can be assumed to take place on a single potential-energy surface, that of the $B$ electronic state. This potential surface is modeled as a sum of pairwise atom-atom interactions, each of them described by a Morse function. The Morse parameters used in the calculations are given in Table I. The potential obtained with these parameters led to a good agreement between experiment and quantum-mechanical calculations in the simu-
TABLE I. Atom-atom Morse potential parameters and masses used in the calculations.

\begin{tabular}{cccc}
\hline \hline & $D_{e}\left(\mathrm{~cm}^{-1}\right)$ & $\alpha\left(a_{0}^{-1}\right)$ & $r_{e}\left(a_{0}\right)$ \\
\hline $\mathrm{Cl}-\mathrm{Cl}$ & 3145.0 & 1.245 & 4.5612 \\
$\mathrm{Cl}-\mathrm{Ne}$ & 39.0 & 0.9525 & 6.9920 \\
$\mathrm{Ne}-\mathrm{Ne}$ & 29.4 & 1.1049 & 5.8412 \\
Atom & $\mathrm{Cl}$ & $\mathrm{Ne}$ & \\
Mass(amu $)$ & 35.4527 & 20.1797 & \\
\hline \hline
\end{tabular}

lation of the $\mathrm{Cl}_{2}-\mathrm{Ne}$ VP dynamics. ${ }^{16,17,19}$ The potential surface employed here is the same as that used in the calculations of Refs. 15 and 22.

\section{B. Initial state}

Prior to carrying out the dynamical simulation the initial state of $\mathrm{Cl}_{2}-\mathrm{Ne}_{2}$ has to be calculated. This initial state corresponds with the ground resonance state of the complex vdW modes associated to the specific $\mathrm{Cl}_{2}$ vibrational level excited in the $B$ electronic state. The $\mathrm{Cl}_{2}-\mathrm{Ne}_{2}$ system is represented in bond coordinates $\left(\mathbf{r}, \mathbf{R}_{\mathbf{1}}, \mathbf{R}_{\mathbf{2}}\right)$, where $\mathbf{r}$ is the vector associated with the $\mathrm{Cl}-\mathrm{Cl}$ bond, and $\mathbf{R}_{\mathbf{1}}, \mathbf{R}_{\mathbf{2}}$ are the vectors between the $\mathrm{Cl}_{2}$ center-of-mass and the two $\mathrm{Ne}$ atoms, respectively. In this representation the Hamiltonian of the system can be expressed as

$$
\begin{aligned}
\hat{H}= & -\hbar^{2}\left\{\frac{\nabla_{\mathbf{r}}^{2}}{2 \mu_{\mathrm{Cl}_{2}}}+\frac{\nabla_{\mathbf{1}}^{2}}{2 \mu_{\mathrm{Cl}_{2}-\mathrm{Ne}}}+\frac{\nabla_{\mathbf{2}}{ }^{2}}{2 \mu_{\mathrm{Cl}_{2}-\mathrm{Ne}}}+\frac{\nabla_{\mathbf{1}} \cdot \nabla_{\mathbf{2}}}{2 m_{\mathrm{Cl}}}\right\} \\
& +V\left(\mathbf{r}, \mathbf{R}_{\mathbf{1}}, \mathbf{R}_{\mathbf{2}}\right),
\end{aligned}
$$

where $\mu_{\mathrm{Cl}_{2}}=m_{\mathrm{Cl}} / 2$ and $\mu_{\mathrm{Cl}_{2}-\mathrm{Ne}}=m_{\mathrm{Ne}} 2 m_{\mathrm{Cl}} /\left(m_{\mathrm{Ne}}+2 m_{\mathrm{Cl}}\right)$ are the reduced masses corresponding to the $\mathrm{Cl}_{2}$ and $\mathrm{vdW}$ modes, respectively. By expressing the operators $\nabla_{\mathbf{r}}, \nabla_{\mathbf{1}}$, and $\nabla_{\mathbf{2}}$ in spherical coordinates $\left[\mathbf{r}=\left(r, \theta_{r}, \phi_{r}\right), \mathbf{R}_{\mathbf{i}}\right.$ $\left.=\left(R_{i}, \theta_{i}, \phi_{i}\right), i=1,2\right]$ the Hamiltonian operator becomes

$$
\begin{aligned}
\hat{H}= & -\frac{\hbar^{2}}{2 \mu_{C l_{2}}} \frac{\partial^{2}}{\partial r^{2}}+\frac{\mathbf{j}^{2}}{2 \mu_{C l_{2}} r^{2}}-\frac{\hbar^{2}}{2 \mu_{C l_{2}-N e}}\left(\frac{\partial^{2}}{\partial R_{1}^{2}}+\frac{\partial^{2}}{\partial R_{2}^{2}}\right) \\
& +\frac{1}{2 \mu_{C l_{2}-N e}}\left(\frac{\mathbf{l}_{\mathbf{1}}^{2}}{R_{1}^{2}}+\frac{\mathbf{l}_{\mathbf{2}}^{2}}{R_{2}^{2}}\right)-\frac{\hbar^{2} \nabla_{\mathbf{1}} \cdot \nabla_{\mathbf{2}}}{2 m_{C l}}+V\left(\mathbf{r}, \mathbf{R}_{\mathbf{1}}, \mathbf{R}_{\mathbf{2}}\right),
\end{aligned}
$$

where $\mathbf{j}, \mathbf{l}_{\mathbf{1}}$, and $\mathbf{l}_{\mathbf{2}}$ are the angular momentum operators associated with $\mathbf{r}, \mathbf{R}_{\mathbf{1}}$, and $\mathbf{R}_{\mathbf{2}}$, respectively (the total angular momentum of the system is $\mathbf{J}=\mathbf{j}+\mathbf{l}_{\mathbf{1}}+\mathbf{l}_{\mathbf{2}}$ ), and

$$
\begin{aligned}
\nabla_{\mathbf{1}} \cdot \nabla_{\mathbf{2}}= & \left(\sin \theta_{1} \sin \theta_{2} \cos \phi+\cos \theta_{1} \cos \theta_{2}\right) \frac{\partial^{2}}{\partial R_{1} \partial R_{2}}+\frac{\cos \theta_{1} \cos \theta_{2} \cos \phi+\sin \theta_{1} \sin \theta_{2}}{R_{1} R_{2}} \frac{\partial^{2}}{\partial \theta_{1} \partial \theta_{2}} \\
& +\frac{\cos \phi}{R_{1} R_{2} \sin \theta_{1} \sin \theta_{2}} \frac{\partial^{2}}{\partial \phi_{1} \partial \phi_{2}}+\frac{\sin \theta_{1} \cos \theta_{2} \cos \phi-\cos \theta_{1} \sin \theta_{2}}{R_{2}} \frac{\partial^{2}}{\partial R_{1} \partial \theta_{2}} \\
& +\frac{\cos \theta_{1} \sin \theta_{2} \cos \phi-\sin \theta_{1} \cos \theta_{2}}{R_{1}} \frac{\partial^{2}}{\partial R_{2} \partial \theta_{1}}-\frac{\sin \theta_{1} \sin \phi}{R_{2} \sin \theta_{2}} \frac{\partial^{2}}{\partial R_{1} \partial \phi_{2}}-\frac{\sin \theta_{2} \sin \phi}{R_{1} \sin \theta_{1}} \frac{\partial^{2}}{\partial R_{2} \partial \phi_{1}} \\
& -\frac{\cos \theta_{1} \sin \phi}{R_{1} R_{2} \sin \theta_{2}} \frac{\partial^{2}}{\partial \theta_{1} \partial \phi_{2}}-\frac{\cos \theta_{2} \sin \phi}{R_{1} R_{2} \sin \theta_{1}} \frac{\partial^{2}}{\partial \theta_{2} \partial \phi_{1}},
\end{aligned}
$$


being $\phi=\phi_{1}-\phi_{2}$.

In the treatment of Ref. 22 a reduced-dimensionality Hamiltonian was assumed

$$
\begin{aligned}
\hat{H}= & -\frac{\hbar^{2}}{2 \mu_{\mathrm{Cl}_{2}}} \frac{\partial^{2}}{\partial r^{2}}-\frac{\hbar^{2}}{2 \mu_{\mathrm{Cl}_{2}-\mathrm{Ne}}}\left(\frac{\partial^{2}}{\partial R_{1}^{2}}+\frac{\partial^{2}}{\partial R_{2}^{2}}\right) \\
& -\frac{\hbar^{2} \cos \phi}{2 m_{\mathrm{Cl}}} \frac{\partial^{2}}{\partial R_{1} \partial R_{2}}+V\left(r, R_{1}, R_{2} ; \phi\right),
\end{aligned}
$$

where the angles $\theta_{1}, \theta_{2}$, and $\phi$ were fixed to $\theta_{1}=\theta_{2}=90^{\circ}$ and $\phi=54^{\circ}$. The initial resonance states were obtained by diagonalizing the Hamiltonian of Eq. (7) using a suitable basis set. ${ }^{22}$ Our approach requires the calculation of the fulldimensional resonance wave function and energy, which involves diagonalization of the full Hamiltonian of Eq. (5). To this purpose we have applied the variational formalism of Villarreal et al., ${ }^{26}$ which will be briefly described in the following.

In order to diagonalize the Hamiltonian of Eq. (5), the first step consists of defining a suitable basis set in a bodyfixed (BF) frame (in which the $z$ axis coincides with the direction of the $\mathbf{r}$ vector). The functions of this basis set are defined as

$$
\begin{aligned}
& \Phi_{l_{1}, l_{2}, L, \Omega, v, m, n}^{J, M}\left(\mathbf{r}, \mathbf{R}_{\mathbf{1}}, \mathbf{R}_{2}\right) \\
& \quad=\phi_{v, m, n}\left(r, R_{1}, R_{2}\right) \mathcal{W}_{l_{1}, l_{2}, L, \Omega}^{J, M}\left(\theta_{1}, \theta_{2}, \phi_{1}, \phi_{2}\right) .
\end{aligned}
$$

The radial function $\phi_{v, m, n}\left(r, R_{1}, R_{2}\right)$ is defined as

$$
\phi_{v, m, n}\left(r, R_{1}, R_{2}\right)=\chi_{v}(r) \xi_{m}\left(R_{1}\right) \xi_{n}\left(R_{2}\right) / r R_{1} R_{2},
$$

where $\chi_{v}(r)$ is an eigenfunction of the $\mathrm{Cl}_{2}$ stretching mode, obtained as a numerical solution of

$$
\left[-\frac{\hbar^{2}}{2 \mu_{C l_{2}}} \frac{\partial^{2}}{\partial r^{2}}+V_{\mathrm{Cl}_{2}}(r)\right] \chi_{v}(r)=E_{v} \chi_{v}(r),
$$

and $\xi_{m}\left(R_{1}\right), \xi_{n}\left(R_{2}\right)$ are vibrational basis functions for the $R_{1}, R_{2}$ coordinates [details on the calculation of $\xi_{m}\left(R_{1}\right)$, $\xi_{n}\left(R_{2}\right)$ are given in Ref. 26].

The angular functions $\mathcal{W}_{l_{1}, l_{2}, L, \Omega}^{J, M}\left(\theta_{1}, \theta_{2}, \phi_{1}, \phi_{2}\right)$ are defined as

$$
\begin{aligned}
& \mathcal{W}_{l_{1}, l_{2}, L, \Omega}^{J, M}\left(\theta_{1}, \theta_{2}, \phi_{1}, \phi_{2}\right) \\
& \quad=\left(\frac{2 J+1}{4 \pi}\right)^{1 / 2} D_{M, \Omega}^{J *}\left(\phi_{r}, \theta_{r}, 0\right) \mathcal{Y}_{l_{1}, l_{2}}^{L, \Omega}\left(\theta_{1}, \theta_{2}, \phi_{1}, \phi_{2}\right),
\end{aligned}
$$

where $D_{M, \omega}^{J *}$ are Wigner rotation matrices ${ }^{27,28}$ which relate the space-fixed (SF) and BF frames corresponding to a total angular momentum $J$ with projections $M$ and $\Omega$ on the $Z_{\mathrm{SF}}$ and $Z_{\mathrm{BF}}$ axes, respectively. The functions $\mathcal{Y}_{l_{1}, l_{2}}^{L, \Omega}\left(\theta_{1}, \theta_{2}, \phi_{1}, \phi_{2}\right)$ are expressed as

$$
\begin{gathered}
\mathcal{Y}_{l_{1}, l_{2}}^{L, \Omega}\left(\theta_{1}, \theta_{2}, \phi_{1}, \phi_{2}\right) \\
=(-1)^{L+\Omega}(2 L+1)^{1 / 2} \sum_{\omega}\left(\begin{array}{ccc}
l_{1} & l_{2} & L \\
-\omega & \omega-\Omega & \omega
\end{array}\right) \\
\times Y_{l_{1}}^{\omega}\left(\theta_{1}, \phi_{1}\right) Y_{l_{2}}^{\Omega-\omega}\left(\theta_{2}, \phi_{2}\right),
\end{gathered}
$$

where $L=l_{1}+l_{2},\left(\begin{array}{c}\ldots \\ \ldots\end{array}\right)$ denotes $3-j$ symbols and $Y_{l_{i}}^{\omega}\left(\theta_{i}, \phi_{i}\right)$ are spherical harmonics. We are interested in states of $\mathrm{Cl}_{2}-\mathrm{Ne}_{2}$ with $J=0$, which implies $M=\Omega=0$, and $\mathbf{L}=-\mathbf{j}$.

The size of the basis set of Eq. (8) can be reduced by taking into account the symmetry properties of the $\mathrm{Cl}_{2}-\mathrm{Ne}_{2}$ Hamiltonian, i.e., the Hamiltonian is invariant under exchange of $\mathbf{R}_{\mathbf{1}}$ and $\mathbf{R}_{\mathbf{2}}$. Thus, one can define new basis functions as linear combinations of those of Eq. (8)

$$
\begin{aligned}
& \Xi_{l_{1}, l_{2}, L, \Omega, v, m, n}^{J, M}\left(\mathbf{r}, \mathbf{R}_{\mathbf{1}}, \mathbf{R}_{\mathbf{2}}\right) \\
& =\left[\frac{1}{2\left(1+\delta_{m n} \delta_{l_{1} l_{2}}\right)}\right]^{1 / 2}\left[\Phi_{l_{1}, l_{2}, L, \Omega, v, m, n}^{J, M}\left(\mathbf{r}, \mathbf{R}_{\mathbf{1}}, \mathbf{R}_{\mathbf{2}}\right)\right. \\
& \left.\quad+\Phi_{l_{2}, l_{1}, L, \Omega, v, n, m}^{J, M}\left(\mathbf{r}, \mathbf{R}_{\mathbf{1}}, \mathbf{R}_{\mathbf{2}}\right)\right] .
\end{aligned}
$$

The Hamiltonian of Eq. (5) is represented in the basis set defined by Eq. (13) and diagonalized. A variety of $\mathrm{Cl}_{2}-\mathrm{Ne}_{2}$ states is obtained,

$$
\begin{aligned}
\Psi_{k}^{0,0}\left(r, R_{1}, R_{2}, \theta_{1}, \theta_{2}, \phi_{1}, \phi_{2}\right) \\
=\sum_{l_{2}, l_{1}, L, v, m, n} A_{k}^{l_{2}, l_{1}, L, 0, v, m, n} \\
\quad \times \Xi_{l_{1}, l_{2}, L, 0, v, m, n}^{0,0}\left(r, R_{1}, R_{2}, \theta_{1}, \theta_{2}, \phi_{1}, \phi_{2}\right),
\end{aligned}
$$

out of which we are interested in the ground resonance state, $k=0$.

In the calculation only one $\chi_{v}(r)$ state was considered in the basis, that corresponding to the vibrational state $v=v^{\prime}$ to which the $\mathrm{Cl}_{2}$ subunit is excited in the complex. Due to the difference of frequencies the $\mathrm{Cl}_{2}$ vibration is well separated from the vdW modes, and inclusion of only one $\chi_{v}(r)$ state suffices, to a good approximation, for the calculation of the ground resonance state of $\mathrm{Cl}_{2}-\mathrm{Ne}_{2}$. The same approximation was made in the calculation of the initial state in Ref. 22. For the other parameters of the basis set we used $l_{1}^{\max }=l_{2}^{\max }$ $=19, L^{\max }=16$, and $m^{\max }=n^{\max }=4$, which led to a set of 12060 basis functions. With this basis set convergence tests showed that the calculated resonance energies are converged within $1 \%-1.5 \%$. These energies are listed in Table II for the different vibrational states $v^{\prime}$ of $\mathrm{Cl}_{2}$ studied $\left(v^{\prime}\right.$ $=7,8,9,11,13)$, and compared with those calculated in Ref. 22. The vibrational energies of $\mathrm{Cl}_{2}, E_{v^{\prime}}$, are also given in the table. Our energies $E_{v}$, are about $10 \mathrm{~cm}^{-1}$ lower than those of Ref. 22, probably due to a slightly different chlorine mass used here.

To the best of our knowledge, the calculation reported here is the first full-dimensional variational calculation of the resonance states of $\mathrm{Cl}_{2}-\mathrm{Ne}_{2}$. Our resonance energies, around $-125 \mathrm{~cm}^{-1}$, are significantly higher than the RDQ ones obtained in Ref. 22 (around $-151 \mathrm{~cm}^{-1}$ ). Our dynamical calculations, carried out at the energies of Table II, sample different regions of the potential surface, and therefore, somewhat different intermode couplings, as compared with those of Ref. 22. In the case of the hybrid quantum-classical calculations Bastida et al. ${ }^{15}$ assumed a value of $-125.3 \mathrm{~cm}^{-1}$ for the resonance energies, and their initial conditions were consistent with this value, which is very close to our calculated energies. 
TABLE II. Calculated full-dimensional (this work) and reduced-dimensionality (Ref. 22) resonance energies relative to the initial $\mathrm{Cl}_{2}$ vibrational energy level $E_{v^{\prime}} . E_{v^{\prime}}=0$ corresponds to separated atoms.

\begin{tabular}{lccccccc}
\hline \hline & $v^{\prime}=7$ & $v^{\prime}=8$ & $v^{\prime}=9$ & $v^{\prime}=10$ & $v^{\prime}=11$ & $v^{\prime}=12$ & $v^{\prime}=13$ \\
\hline$E_{v^{\prime}}\left(\mathrm{cm}^{-1}\right)$ & -1511.1 & -1338.0 & -1175.4 & -1023.3 & -881.8 & -750.8 & -630.3 \\
$E_{\text {res }}^{a}\left(\mathrm{~cm}^{-1}\right)^{\mathrm{a}}$ & -125.67 & -125.43 & -125.24 & & -123.97 & & -123.67 \\
$E_{\text {res }}^{b}\left(\mathrm{~cm}^{-1}\right)^{\mathrm{b}}$ & -152.3 & -152.13 & -151.97 & -151.26 & -151.48 & -151.15 & -150.67 \\
\hline \hline
\end{tabular}

${ }^{\mathrm{a}}$ This work.

${ }^{\mathrm{b}}$ Reference 22.

It should be noted that the experimental vdW bond energy was determined to be between 145.6 and $148.6 \mathrm{~cm}^{-1}$, from measurements of the highest rotational state of $\mathrm{Cl}_{2}$ produced by $\mathrm{Cl}_{2}(B, v=9)-\mathrm{Ne}_{2} \rightarrow \mathrm{Cl}_{2}(B, v=8, j)+2 \mathrm{Ne}$, being the maximum $j$ detected $j_{\max }=10$. This energy is about $20 \mathrm{~cm}^{-1}$ higher than our variationally calculated bond energies $\approx 125 \mathrm{~cm}^{-1}$. It was discussed in Ref. 15 that the experimental vdW bond energy could be overestimated due to difficulties in detecting higher $j$ states because of their small population. Deficiencies in the present potential surface could also be responsible of the discrepancy.

Now, in order to obtain the initial state to be used in our RDQ dynamical calculations, the full-dimensional resonance state $\Psi_{k=0}^{0,0}$ is factorized as follows:

$$
\begin{aligned}
& \Psi_{k=0, v^{\prime}}^{0,0}\left(r, R_{1}, R_{2}, \theta_{1}, \theta_{2}, \phi_{1}, \phi_{2}\right) \\
& \quad \simeq \psi_{v^{\prime}}\left(r, R_{1}, R_{2}\right) \mathcal{F}_{v^{\prime}}\left(\theta_{1}, \theta_{2}, \phi_{1}, \phi_{2}\right),
\end{aligned}
$$

and $\psi_{v^{\prime}}\left(r, R_{1}, R_{2}\right)$ is adopted as the initial state associated with the $\mathrm{Cl}_{2}$ vibrational state $v^{\prime}$. We note that the initial state $\psi_{v^{\prime}}\left(r, R_{1}, R_{2}\right)$ is expected to be more accurate and realistic than that obtained by diagonalizing the Hamiltonian of Eq. (7).

\section{Reduced-dimensionality Hamiltonian}

Our RDQ dynamical model considers only the radial degrees of freedom $r, R_{1}$, and $R_{2}$. Thus, a reduceddimensionality Hamiltonian for these degrees of freedom is to be obtained from the full-dimensional one of Eq. (5). A simple and systematic approach to obtain such a Hamiltonian consists of averaging the full Hamiltonian of Eq. (5) over the angular part of the full-dimensional initial state, $\mathcal{F}_{v^{\prime}}\left(\theta_{1}, \theta_{2}, \phi_{1}, \phi_{2}\right)$,

$$
\begin{aligned}
\hat{H}_{v^{\prime}}^{\text {red }}\left(r, R_{1}, R_{2}\right)= & \left\langle\mathcal{F}_{v^{\prime}}\left(\theta_{1}, \theta_{2}, \phi_{1}, \phi_{2}\right)\right. \\
& \left.\times|\hat{H}| \mathcal{F}_{v^{\prime}}\left(\theta_{1}, \theta_{2}, \phi_{1}, \phi_{2}\right)\right\rangle .
\end{aligned}
$$

The zero-point energy of the angular modes is incorporated into the reduced Hamiltonian $\hat{H}_{v^{\prime}}^{\text {red }}\left(r, R_{1}, R_{2}\right)$ in a natural way by the averaging of Eq. (16). Now the dynamics governed by $\hat{H}_{v^{\prime}}^{\text {red }}\left(r, R_{1}, R_{2}\right)$ takes place at the full-dimensional energy of the $\mathrm{Cl}_{2}-\mathrm{Ne}_{2}$ complex. It should be noted that the factorization of the resonance state [Eq. (15)] breaks the couplings between the stretching and the bending modes. This causes a slight variation (about 2\% in the present case) of the resonance energy associated with the factorized state, as compared with the energy of the coupled resonance state calcu- lated variationally. Therefore the total energy of the RDQ dynamical calculation deviates slightly from the correct fulldimensional energy by this small variation.

In addition to incorporating in a systematic way the bending zero-point energy, the model Hamiltonian $\hat{H}_{v^{\prime}}^{\text {red }}\left(r, R_{1}, R_{2}\right)$ has another important advantage. It is related to the fact that all the terms appearing in the full Hamiltonian of Eq. (5) are retained in $\hat{H}_{v^{\prime}}^{\text {red }}\left(r, R_{1}, R_{2}\right)$. Indeed, leaving aside $V\left(\mathbf{r}, \mathbf{R}_{\mathbf{1}}, \mathbf{R}_{\mathbf{2}}\right)$, the kinetic-energy operator of $\hat{H}$ consists of 15 terms, which are conserved in $\hat{H}_{v^{\prime}}^{\text {red }}\left(r, R_{1}, R_{2}\right)$ with the angular dependence of each term averaged over $\left|\mathcal{F}_{v^{\prime}}\right|^{2}$. In contrast, the model Hamiltonian of Eq. (7) only conserves four kinetic-energy terms out of the 15 ones of the full Hamiltonian (all the kinetic-energy terms with an angular dependence are neglected). The more flexible model Hamiltonian $\hat{H}_{v^{\prime}}^{\text {red }}\left(r, R_{1}, R_{2}\right)$ includes all the coupling terms between the modes $R_{1}$ and $R_{2}$, and therefore it is expected to provide a more realistic description of the energy transfer dynamics.

\section{Dynamics simulations}

The VP dynamics of $\mathrm{Cl}_{2}-\mathrm{Ne}_{2}$ is simulated by solving the time-dependent Schrödinger equation

$$
i \hbar \frac{\partial \psi\left(r, R_{1}, R_{2}, t\right)}{\partial t}=\hat{H}_{v^{\prime}}^{\mathrm{red}} \psi\left(r, R_{1}, R_{2}, t\right),
$$

starting from the initial state $\psi\left(r, R_{1}, R_{2}, t=0\right)$ $=\psi_{v^{\prime}}\left(r, R_{1}, R_{2}\right)$, and assuming $J=0$. The wave packet $\psi\left(r, R_{1}, R_{2}, t\right)$ is expanded on the vibrational states $\chi_{v}(r)$ of $\mathrm{Cl}_{2}$ as

$$
\psi\left(r, R_{1}, R_{2}, t\right)=\sum_{v} C_{v}\left(R_{1}, R_{2}, t\right) \chi_{v}(r) e^{-i E_{v} t / \hbar},
$$

and introducing this expression of $\psi\left(r, R_{1}, R_{2}, t\right)$ in Eq. (17) leads to a set of time-dependent coupled equations for the different $C_{v}\left(R_{1}, R_{2}, t\right)$ packets, which are the ones actually solved. Four terms in the expansion of Eq. (18), $v=v^{\prime}, v^{\prime}$ $-1, v^{\prime}-2$, and $v^{\prime}-3$ are sufficient to describe the VP dynamics of $\mathrm{Cl}_{2}-\mathrm{Ne}_{2}$ (in Ref. 22 only three terms, $v=v^{\prime}, v^{\prime}$ -1 , and $v^{\prime}-2$ were considered in the same expansion).

In order to propagate the $C_{v}\left(R_{1}, R_{2}, t\right)$ packets, they are represented on a uniform grid in the $R_{1}$ and $R_{2}$ coordinates. We adopted the grid parameters of Ref. 22, i.e., $R_{0}$ $=4.0$ a.u., $\Delta R=0.25$ a.u., and the number of grid points $N_{R}=128$. The time propagation of the $C_{v}\left(R_{1}, R_{2}, t\right)$ packets is carried out by means of the Chebychev polynomial expan- 
sion method ${ }^{29}$ with a time step $\Delta t=0.01$ ps. All the Hamiltonian operations on $C_{v}\left(R_{1}, R_{2}, t\right)$ involving kinetic-energy terms are performed using fast Fourier transform (FFT) techniques.

The wave packet is absorbed before it reaches the edges of the grid in the $R_{1}$ and $R_{2}$ coordinates. Absorption is carried out after each time step $\Delta t$ by multiplying each packet $C_{v}\left(R_{1}, R_{2}, t\right)$ by the function $a\left(R_{1}\right) a\left(R_{2}\right)$

$$
C_{v}^{a}\left(R_{1}, R_{2}, t\right)=a\left(R_{1}\right) a\left(R_{2}\right) C_{v}\left(R_{1}, R_{2}, t\right),
$$

where, following Ref. 22, $a\left(R_{i}\right), i=1,2$ is defined as

$a\left(R_{i}\right)=\exp \left[-\alpha\left(R_{i}-R_{\mathrm{abs}}\right)^{2}\right], \quad R_{\mathrm{abs}} \leqslant R_{i} \leqslant R_{\max }$,

$a\left(R_{i}\right)=1, \quad R_{i}<R_{\mathrm{abs}}$,

and the absorption parameters $\alpha=0.20$ a.u. and $R_{\text {abs }}$ $=25.0$ a.u. are taken from Ref. 22. After each absorption step, propagation continues by replacing $C_{v}$ by $C_{v}^{a}$. For the analysis of product distributions it is considered that for distances $R_{1}, R_{2}>R_{c}=15$ a.u. (as in Ref. 22) the vdW bonds are effectively broken.

\section{E. Resonance lifetimes and $\mathrm{Cl}_{2}$ vibrational distributions}

The observables of dynamical interest in the VP process of $\mathrm{Cl}_{2}-\mathrm{Ne}_{2}$ are typically the resonance decay lifetime and the vibrational and rotational distributions of the $\mathrm{Cl}_{2}$ fragment. Rotational distributions cannot be extracted from our model calculations since the angular modes are not considered in the dynamics. Resonance lifetimes and $\mathrm{Cl}_{2}$ vibrational distributions can be obtained, and in the following it is described how they are calculated.

Resonance decay lifetimes can be extracted either from the square of the wave packet autocorrelation function $P(t)$ $=|\langle\psi(0) \mid \psi(t)\rangle|^{2}$ or from the survival probability of the resonance state $P(t)=\left\langle C_{v^{\prime}}\left(R_{1}, R_{2}, t\right) \mid C_{v^{\prime}}\left(R_{1}, R_{2}, t\right)\right\rangle$. By fitting either of the above definitions of the decay curve to an exponential law

$$
P(t) \simeq e^{-t / \tau},
$$

[or equivalently, by fitting $\ln P(t)$ to the straight line $-t / \tau$ ], the resonance lifetime $\tau$ is obtained. The assumption of a single exponential form for the decay curve made in Eq. (21) implies that the initial state is a pure, isolated resonance state. Our initial state is a good approximation to this situation.

We chose to calculate the resonance decay curve as the square of the wave packet autocorrelation function $C(t)$. The advantage of the autocorrelation function is that by propagating the wave packet until a final time $t_{f}$, one can obtain $C(t)$ until a time $2 t_{f}$, since it has been shown ${ }^{30}$ that

$$
\begin{aligned}
C(t) & =\langle\psi(0) \mid \psi(t)\rangle \\
& =\left\langle e^{i \hat{H} t / 2 \hbar} \psi(0) \mid e^{-i \hat{H} t / 2 \hbar} \psi(0)\right\rangle \\
& =\left\langle\psi^{*}(t / 2) \mid \psi(t / 2)\right\rangle .
\end{aligned}
$$

The use of the expression of Eq. (22) poses some difficulties due to the absorption of the wave packet, although they can be overcome.
Following the notation of Pernot and Lester, ${ }^{31}$ after the first absorption step the wave packet splits in two parts

$$
\psi\left(t_{1}\right)=\left(1-f_{p}\right) \psi\left(t_{1}\right)+f_{p} \psi\left(t_{1}\right)=\psi_{I}^{1}\left(t_{1}\right)+\psi_{P}^{1}\left(t_{1}\right),
$$

being $f_{p}\left(R_{1}, R_{2}\right)=a\left(R_{1}\right) a\left(R_{2}\right), t_{1}=t+\Delta t$, and $\psi_{P}^{1}$ the part of wave packet which is absorbed. After the second absorption step a further split takes place

$$
\begin{aligned}
\psi\left(t_{2}\right) & =\psi_{I}^{2}\left(t_{2}\right)+\psi_{P}^{2}\left(t_{2}\right)+\hat{U}(\Delta t) \psi_{P}^{1}\left(t_{1}\right) \\
& =\psi_{I}^{2}\left(t_{2}\right)+\psi_{P}^{2}\left(t_{2}\right)+\psi_{P}^{1}\left(t_{2}\right),
\end{aligned}
$$

where $\hat{U}$ is the time evolution operator and $t_{2}=t+2 \Delta t$. After $n$ absorption steps at times $t_{1}, t_{2}, \ldots, t_{n}=t+n \Delta t$, the wave packet may be expressed as

$$
\begin{aligned}
\psi\left(t_{n}\right) & =\psi_{I}^{n}\left(t_{n}\right)+\sum_{i=1}^{n} \hat{U}[(n-i) \Delta t] \psi_{P}^{i}\left(t_{i}\right) \\
& =\psi_{I}^{n}\left(t_{n}\right)+\sum_{i=1}^{n} \psi_{P}^{i}\left(t_{n}\right) .
\end{aligned}
$$

Thus, after $n$ absorption splittings, the wave packet autocorrelation function can be expressed as

$$
\begin{aligned}
C\left(2 t_{n}\right)= & \left\langle\psi^{*}\left(t_{n}\right) \mid \psi\left(t_{n}\right)\right\rangle \\
= & \left\langle\psi_{I}^{n *}\left(t_{n}\right) \mid \psi_{I}^{n}\left(t_{n}\right)\right\rangle+2 \operatorname{Re}\left\langle\psi_{I}^{n *}\left(t_{n}\right) \mid \psi_{P}^{n}\left(t_{n}\right)\right\rangle \\
& +\left\langle\psi_{P}^{n *}\left(t_{n}\right) \mid \psi_{P}^{n}\left(t_{n}\right)\right\rangle+\sum_{i=1}^{n-1}\left[\left\langle\psi_{P}^{i *}\left(t_{n}\right) \mid \psi_{P}^{i}\left(t_{n}\right)\right\rangle\right. \\
& +2 \operatorname{Re}\left\langle\psi_{I}^{n *}\left(t_{n}\right) \mid \psi_{P}^{i}\left(t_{n}\right)\right\rangle \\
& \left.+\sum_{j=i+1}^{n} 2 \operatorname{Re}\left\langle\psi_{P}^{j *}\left(t_{n}\right) \mid \psi_{P}^{i}\left(t_{n}\right)\right\rangle\right] .
\end{aligned}
$$

The three first terms of the right-hand-side (rhs) of Eq. (26) can be easily calculated after each absorption splitting of the wave packet, but the calculation of the remaining terms is much more complicated. The reason is that in order to calculate the terms inside the sums in Eq. (26), the wave packet pieces $\psi_{P}^{i}$ absorbed at a time $t_{i}<t_{n}$ have to be propagated until $t_{n}$. We decided to calculate the autocorrelation function by considering only the three first terms of the rhs of Eq. (26), and neglecting the other terms. A comparison of this autocorrelation function with that calculated as $C(t)$ $=\langle\psi(0) \mid \psi(t)\rangle$ in the time interval of the wave packet propagation, $\left[0, t_{f}\right]$, showed an excellent agreement (the two functions are indistinguishable) for all the resonance states of $\mathrm{Cl}_{2}-\mathrm{Ne}_{2}$ studied. Since the initial wave packet $\psi(0)$ is localized in the interaction region, the autocorrelation function calculated as $C(t)=\langle\psi(0) \mid \psi(t)\rangle$ is not affected by the absorption of the wave packet $\psi(t)$ in the asymptotic region. Therefore, the good agreement between this $C(t)$ and that calculated with Eq. (26) implies that the contribution of the terms neglected in the expression of Eq. (26) is very small.

Actually, the above result is not surprising. The wave packet pieces $\psi_{I}^{n}\left(t_{n}\right)$ and $\psi_{P}^{i}\left(t_{n}\right)$ with $i \leqslant n-1$ are located in different spatial regions, and their mutual overlapings and, therefore, the terms of the type $\operatorname{Re}\left\langle\psi_{I}^{n *}\left(t_{n}\right) \mid \psi_{P}^{\dot{j}}\left(t_{n}\right)\right\rangle$ are expected to be very small. A similar argument holds for the 
pieces $\psi_{P}^{j}\left(t_{n}\right)$ and $\psi_{P}^{i}\left(t_{n}\right)$ with $j \neq i$ and the terms $\operatorname{Re}\left\langle\psi_{P}^{j *}\left(t_{n}\right) \mid \psi_{P}^{j}\left(t_{n}\right)\right\rangle$. It is less obvious from spatial and overlaping considerations that the terms $\left\langle\psi_{P}^{i *}\left(t_{n}\right) \mid \psi_{P}^{i}\left(t_{n}\right)\right\rangle$ with $i$ $\leqslant n-1$ are small. Probably they have different phases, leading to destructive interference between them and to a small value of the sum $\sum_{i=1}^{n-1}\left\langle\psi_{P}^{i *}\left(t_{n}\right) \mid \psi_{P}^{i}\left(t_{n}\right)\right\rangle$.

Obtaining the vibrational distributions is a more involved task than calculating the resonance lifetimes, as discussed in Ref. 22. The quantity of interest is the probability $P_{v_{f}}$ of $\mathrm{Cl}_{2}\left(v_{f}\right)$ products in $v_{f}=v^{\prime}-1, v^{\prime}-2, v^{\prime}-3$, after the fragmentation of the two vdW bonds. One of the main difficulties to calculate these probabilities arises, again, from the absorption procedure. Specifically, in the sequential dissociation pathway of Eq. (1) the fragments $\mathrm{Ne}+\mathrm{Cl}_{2}-\mathrm{Ne}$ are produced after breaking of the first $\mathrm{vdW}$ bond. The triatomic fragment will undergo subsequent dissociation into $\mathrm{Ne}+\mathrm{Cl}_{2}$. However, lifetimes for this second dissociation step are typically twice as long as those for the first one, and the wave packet components corresponding to $\mathrm{Ne}+\mathrm{Cl}_{2}-\mathrm{Ne}$ fragments may be absorbed before $\mathrm{Cl}_{2}-\mathrm{Ne}$ dissociates. Once this wave packet intensity is absorbed it is not propagated further, and its contribution to the probability of $\mathrm{Cl}_{2}\left(v_{f}\right)$ is lost. This leads to underestimate the probability of the vibrational channels producing $\mathrm{Cl}_{2}\left(v_{f}\right)$ with $v_{f}=v^{\prime}-2, v^{\prime}-3$.

Le Quéré and Gray designed a model ${ }^{22}$ which estimates and includes the contributions of the $\mathrm{Ne}+\mathrm{Cl}_{2}-\mathrm{Ne}$ components absorbed to the different $P_{v_{f}}$ probabilities. Such a model is approximate and rather involved, as recognized by the authors in Ref. 22. Our calculation of $P_{v_{f}}$ is similar to that of Ref. 22, although simpler in the sense that we do not consider the possible contributions of the absorbed $\mathrm{Ne}+\mathrm{Cl}_{2}(v)-\mathrm{Ne}$ wave packet components. Thus we calculate $P_{v_{f}}$ by accumulating probability in the region of the products $2 \mathrm{Ne}+\mathrm{Cl}_{2}\left(v_{f}\right)$, including the absorption region. This product region, denoted by $P$ in the following, is that for which the two vdW bonds can be considered broken, i.e., $R_{1}, R_{2}$ $>R_{c}$. Following Pernot and Lester, ${ }^{31}$ and similarly as in Ref. 22 , the probability of products $2 \mathrm{Ne}+\mathrm{Cl}_{2}(v)$ can be calculated at a given time $t_{n}$ as

$$
\begin{aligned}
P_{v}^{P}\left(t_{n}\right)= & \left\langle C_{v}^{a}\left(t_{n}\right) \mid C_{v}^{a}\left(t_{n}\right)\right\rangle_{P} \\
& +\sum_{i=1}^{n}\left[\left\langle C_{v}\left(t_{i}\right)-C_{v}^{a}\left(t_{i}\right) \mid C_{v}\left(t_{i}\right)-C_{v}^{a}\left(t_{i}\right)\right\rangle_{P}\right. \\
& \left.+2 \operatorname{Re}\left\langle C_{v}^{a}\left(t_{i}\right) \mid C_{v}\left(t_{i}\right)-C_{v}^{a}\left(t_{i}\right)\right\rangle_{P}\right],
\end{aligned}
$$

where integration is carried out over $R_{1}$ and $R_{2}$ in the region $P$, and the terms in the sum $\Sigma_{i}$ correspond to the probability absorbed in that region.

In our finite-time propagation, at final time $t_{f}$ the sum of the probabilities $P_{v}^{P}\left(t_{f}\right)$ over all the vibrational channels is not unity, as it should be for an ideally long propagation without absorption. At time $t_{f}$ there is wave packet intensity in the interaction region corresponding to complexes which still did not dissociate to $\mathrm{Cl}_{2}+2 \mathrm{Ne}$ (or $\mathrm{Ne}_{2}$ ). Therefore, the absolute probabilities $P_{v_{f}}^{P}\left(t_{f}\right)$ calculated with Eq. (27) are not indicative of the final values $P_{v_{f}}^{P}(t=\infty)$.
However, an extremely costly long propagation can be avoided in order to obtain reliable estimates of the final vibrational probabilities. It can be achieved by monitoring the time evolution of the relative or normalized probabilities

$$
P_{v_{f}}^{\text {norm }}(t)=\frac{P_{v_{f}}^{P}(t)}{\sum_{v} P_{v}^{P}(t)} .
$$

As discussed in Ref. 22, in $\mathrm{BC}-\mathrm{Rg}_{2} \mathrm{vdW}$ systems, similarly as in $\mathrm{BC}-\mathrm{Rg}$ complexes, the form of the product distributions is essentially determined by the short-time dynamics. The underlying idea is that after enough time (typically a few ps), all the dissociation mechanisms contributing with probability to a given final vibrational channel $v_{f}$, are operating. As we shall see in Sec. III B, at short times the probabilities $P_{v_{f}}^{\text {norm }}(t)$ oscillate with time due to the gradual incorporation of the different dissociation mechanisms. Once all the mechanisms are incorporated, they operate in the same way for further times, producing a stabilization and convergence of $P_{v_{f}}^{\text {norm }}(t)$ to a fixed value. Such values provide the estimates for the final vibrational probabilities of the $\mathrm{Cl}_{2}\left(v_{f}\right)$ fragment.

\section{RESULTS AND DISCUSSION}

\section{A. Resonance lifetimes}

The decay of five resonance states of $\mathrm{Cl}_{2}-\mathrm{Ne}_{2}$, those corresponding with $v^{\prime}=7,8,9,11,13$ of $\mathrm{Cl}_{2}$, has been simulated. Depending on the vibrational state excited the wave packet propagation was carried out until different final times, being $t_{f}=10 \mathrm{ps}$ for $v^{\prime}=13, t_{f}=13 \mathrm{ps}$ for $v^{\prime}=11$, and $t_{f}$ $=15 \mathrm{ps}$ for $v^{\prime}=7,8,9$. The corresponding decay curves are shown in Fig. 1, along with the fits to a single exponential function, Eq. (21).

As seen from Fig. 1, a single exponential law fits well the calculated decay curves. Small undulations in the decay curves are indicative that the initial states are not a pure, isolated resonance, and they include small contributions of other states. Such contributions are originated in the factorization of the initial resonance state. First, in the calculation of the resonance state $\Psi_{k=0, v^{\prime}}^{0,0}$ only one $\chi_{v^{\prime}}(r)$ state is included in the basis set [Eq. (14)], which in practice means that the $r$ dependence is factorized in the initial state. Second, this initial state is further factorized as a product of a radial part and an angular part [Eq. (15)]. The factorized initial state is no longer a pure resonance state, but a mix of states in which the resonance of interest is the dominant state (with a weight close to 100\%). The increasing intensity of the undulations in the decay curves as $v^{\prime}$ decreases indicates that the couplings between the factorized modes become stronger. In fact, upon the factorization of Eq. (15), the small variation of the resonance energy increases from $1.9 \%$ for $v^{\prime}=13$ to $2.2 \%$ for $v^{\prime}=7$, which is an additional indication of the increasing intensity of the couplings between radial and angular modes as $v^{\prime}$ decreases.

From the fits of Fig. 1 the resonance decay lifetimes are obtained. These lifetimes are listed in Table III along with the experimental ones and those previously calculated in 


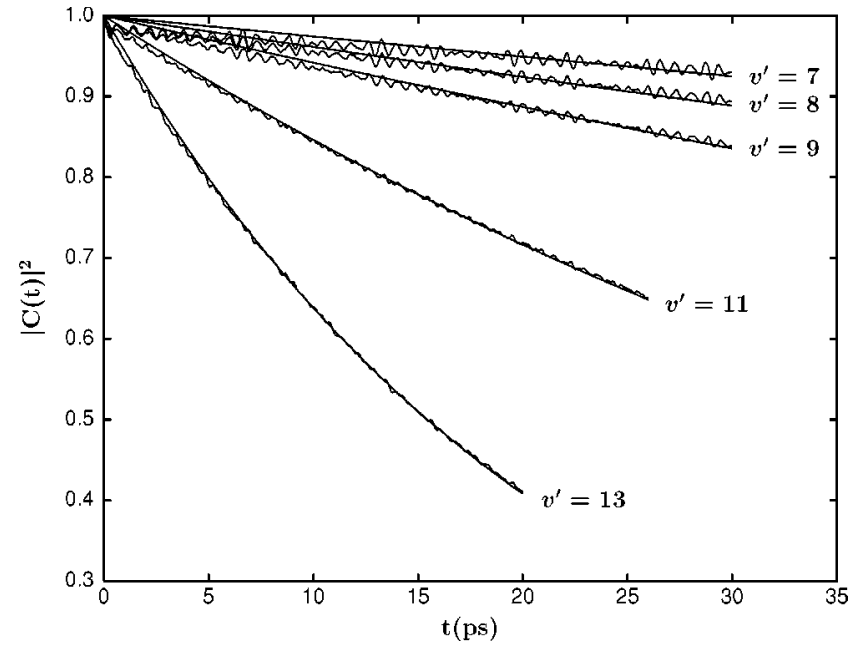

FIG. 1. Decay curves calculated from the autocorrelation function $C(t)$ for the five initial resonance states studied vs time. An exponential fit [Eq. (21)] to each decay curve is also shown.

Refs. 15 and 22. For a better comparison all the lifetimes are plotted in Fig. 2 versus the initial vibrational quantum number $v^{\prime}$.

The lifetimes calculated with our model are higher than the experimental and previously calculated lifetimes by about a factor of 2. Despite the discrepancy between our lifetimes and those calculated by Le Quéré and Gray, the behavior of the lifetimes with $v^{\prime}$ is very similar in both calculations. The trend observed experimentally is an increase of the lifetime with decreasing $v^{\prime}$ following nearly a straight line. This trend is found in both our RDQ model and that of Ref. 22 for the higher $v^{\prime}$ states. As $v^{\prime}$ decreases, the two RDQ models gradually deviate from the experimental behavior (the deviation of our model is higher), predicting a faster increase of the lifetime. We believe that this deviation is caused by an increasing influence of the bending modes neglected on the calculated lifetimes as $v^{\prime}$ decreases. This increasing influence of the angular modes with decreasing $v^{\prime}$ would be due to the increasing intensity of the couplings between the stretching and bending modes discussed above. In this sense we note that only the hybrid quantum-classical results, ${ }^{15}$ which consider all the modes in a coupled fashion, are able to reproduce the experimental trend of the lifetimes in all the range of vibrational excitations, $v^{\prime}=7-13$.

The differences between the present lifetimes and those of Ref. 22 are apparently surprising, taking into account that the same potential surface is used and the two models are not dramatically different. Such differences are essentially caused by including the zero-point energy of the bending modes in our model Hamiltonian. In Ref. 25 the VP lifetimes of $\mathrm{I}_{2}-\mathrm{Ne}_{2}$ were calculated with a RDQ model with and without including the zero-point energy of the neglected angular modes. It was found that including this zero-point energy has the effect of increasing substantially the value of the lifetimes obtained. Our present lifetimes show the same effect when compared with those of Ref. 22.

Our model thus provides a systematic upper limit for the resonance lifetime. The difference between the calculated lifetime and the experimental one is essentially caused by the
TABLE III. Experimental and calculated decay lifetimes (in ps).

\begin{tabular}{rcccc}
\hline \hline$v^{\prime}$ & Experiment $^{\mathrm{a}}$ & This work & $\mathrm{RDQ}^{\mathrm{b}}$ & Hybrid $\mathrm{q} / \mathrm{c}^{\mathrm{c}}$ \\
\hline 13 & 9.5 & 22.3 & 10.5 & 12.2 \\
12 & & & 18.9 & 23.1 \\
11 & 44.4 & 60.0 & 29.7 & 41.2 \\
10 & & & 50.1 & 55.6 \\
9 & & 167.0 & 83.1 & 106.8 \\
8 & 123.7 & 254.0 & 139.0 & 130.5 \\
7 & & 385.0 & 237.0 & \\
\hline \hline
\end{tabular}

${ }^{\mathrm{a}}$ Reference 5 .

${ }^{\mathrm{b}}$ Reference 22.

${ }^{\mathrm{c}}$ Reference 15 .

effect of those modes not considered in the model (provided that the potential surface is reasonably accurate). Upon gradual inclusion of the neglected modes in our model, it is expected that the calculated lifetimes will decrease and converge towards the experimental values. Indeed, relaxing our model and including more degrees of freedom, potential acceptors of energy, will produce a faster resonance decay, leading to lower lifetimes. In RDQ models in which the zeropoint energy of the neglected modes is not included, the calculated lifetimes can be either above or below the experimental values. ${ }^{22,25}$ In some cases the lifetimes calculated with these models will agree better with the experimental ones than those obtained with a model (like the present one) where the full resonance energy is considered. This is the situation of $\mathrm{Cl}_{2}-\mathrm{Ne}_{2}$, where the lifetimes of Ref. 22 are closer to the experimental values than our lifetimes. In other cases the result is the opposite, as for the $\mathrm{I}_{2}-\mathrm{Ne}_{2}$ complex ${ }^{25}$ where inclusion of the neglected zero-point energy improved the agreement with experiment.

\section{B. Vibrational distributions}

Vibrational distributions of the $\mathrm{Cl}_{2}\left(v_{f}\right)$ fragment are calculated by means of Eqs. (27) and (28), as discussed in Sec. IIIE. The time evolution of the normalized (or relative) vibrational populations $P_{v_{f}}^{\text {norm }}(t)\left(v_{f}=v^{\prime}-1, v^{\prime}-2, v^{\prime}-3\right)$ is shown in Fig. 3 for three of the resonance states studied. As

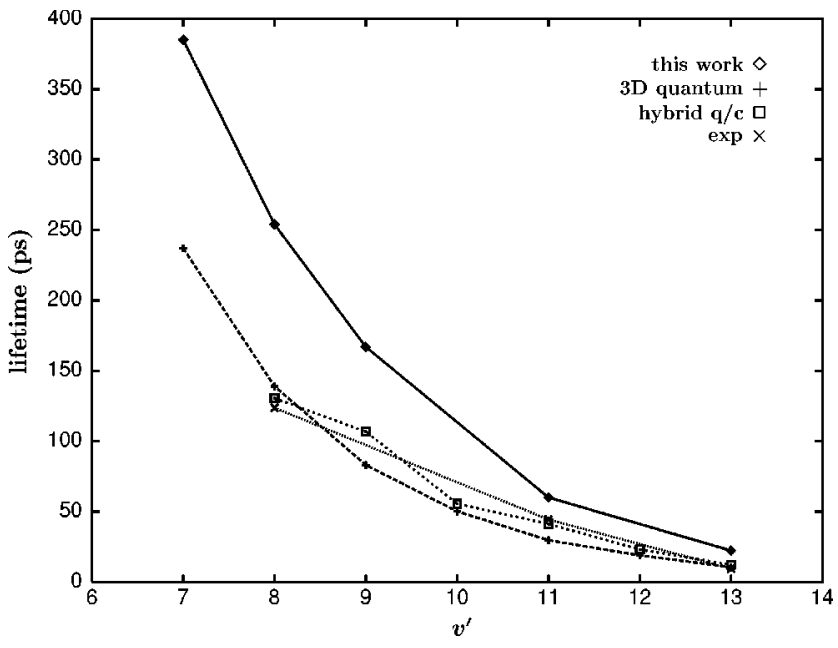

FIG. 2. Plot of the calculated and experimental resonance lifetimes presented in Table III vs the initial $\mathrm{Cl}_{2}$ vibrational level $v^{\prime}$. 
anticipated in Sec. III E, Fig. 3 shows that the different probabilities $P_{v_{f}}^{\mathrm{norm}}(t)$ oscillate for the first ps, and then they stabilize and converge to a fixed value.

The short-time oscillations are due to the gradual population and competition of the different dissociation channels, namely loss of one vibrational quantum $\left(v^{\prime}-1\right)$, of two quanta $\left(v^{\prime}-2\right)$, and of three quanta $\left(v^{\prime}-3\right)$. The lifetime of the process $\Delta v^{\prime}=-1$ is the lowest one as compared with $\Delta v^{\prime}=-2$ and $\Delta v^{\prime}=-3$, which have associated increasingly higher lifetimes, respectively. Therefore, $v^{\prime}-1$ is the first channel being populated. After the $v^{\prime}-1$ channel is populated, population flows sequentially from $v^{\prime}-1$ to $v^{\prime}$ -2 , and from this channel to $v^{\prime}-3$ (direct population of $v^{\prime}-2$ and $v^{\prime}-3$ through $\Delta v^{\prime}=-2$ and $\Delta v^{\prime}=-3$ transitions, respectively, are very unlikely and therefore very slow processes). This sequential population of the three vibrational channels gives rise to the short-time oscillations (e.g., a maximum of $v^{\prime}-1$ at a given time becomes a minimum after some delay and produces a maximum in $v^{\prime}-2$, and similarly for $v^{\prime}-2$ and $v^{\prime}-3$ ).

It takes a few ps for all the processes contributing to populate a given vibrational channel to adjust between themselves, and to produce an average velocity of population of that vibrational channel. Once such an average velocity of population is reached (after $2-5$ ps for all the vibrational states studied) the oscillations cease and the vibrational populations gradually stabilize. After some additional period of time, typically much smaller than the resonance lifetime as shown by Fig. 3, the vibrational populations converge to the final value.

The final vibrational populations of $\mathrm{Cl}_{2}$ in the channels $v^{\prime}-1, v^{\prime}-2$, and $v^{\prime}-3$ are listed in Table IV for the five resonance states investigated. The experimental and previously calculated vibrational distributions are also included in the table for the sake of comparison. The $v^{\prime}-3$ channel was weakly observed experimentally, and no quantitative measurements of the populations have been reported. For this vibrational channel our model predicts similar populations to those obtained with the model of Ref. 22, which are substantially smaller than the $v^{\prime}-3$ populations calculated with the hybrid quantum-classical method. ${ }^{15}$ The two RDQ results agree better with the experimental findings. However, as discussed in Ref. 15, both RDQ models are likely to underestimate the probability of the $v^{\prime}-3$ channel, related to IVR processes, as a result of neglecting the angular modes, which typically act as energy-acceptor, bath modes.

Regarding the $v^{\prime}-1$ and $v^{\prime}-2$ populations, the experimental results for $v^{\prime} \geqslant 10$ (not shown in Table IV) are that $100 \%$ of the population corresponds to the $v^{\prime}-2$ channel. Our results for these channels agree well with those of Le Quéré and Gray ${ }^{22}$ only for $v^{\prime}=13$. For $v^{\prime}<13$ our model predicts significantly larger populations in $v^{\prime}-1$ (and therefore, significantly smaller populations in $v^{\prime}-2$ ). The present results for the $v^{\prime}-1$ and $v^{\prime}-2$ populations are closer to those of Bastida et al. ${ }^{15}$ (although our $v^{\prime}-1$ populations are higher), and to the experimental values, at least for $v^{\prime}<10$. Our results indicate that sequential dissociation through the $v^{\prime}-2$ channel is dominant for $v^{\prime} \geqslant 9$, in agreement with experiment and with the previous calculations. For $v^{\prime}<9$ the
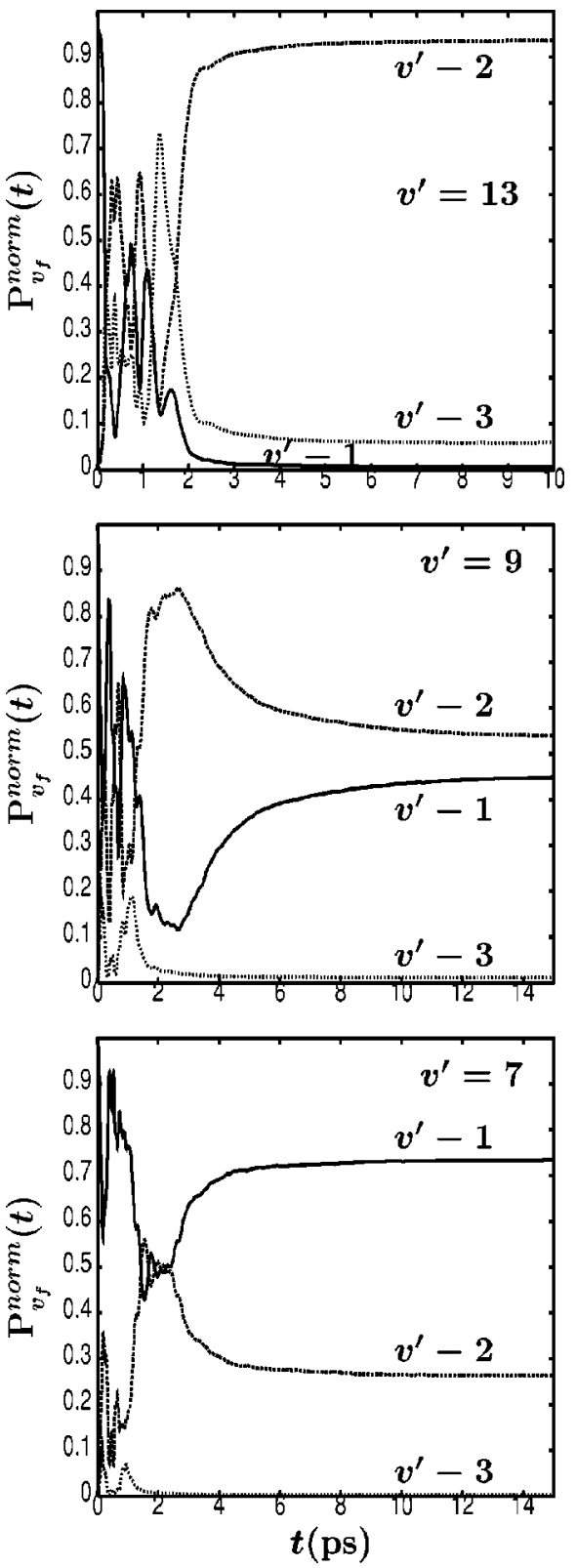

FIG. 3. Time evolution of the normalized vibrational populations of the $\mathrm{Cl}_{2}\left(v_{f}\right)$ fragment $\left(v_{f}=v^{\prime}-1, v^{\prime}-2, v^{\prime}-3\right)$ for three initial $\mathrm{Cl}_{2}$ vibrational levels $v^{\prime}$. See the text for details.

situation is inverted, and now direct dissociation through the $v^{\prime}-1$ channel becomes dominant, also in agreement with the available experimental data. We stress that the calculations of Refs. 15 and 22 do not predict dominance of the $v^{\prime}-1$ channel for any of the $v^{\prime}$ states studied.

We believe that our $v^{\prime}-1$ populations are overestimated to some extent due to the contribution of two factors. One factor is the absence of the energy-acceptor angular modes in our model. In the present model, when $\mathrm{Cl}_{2}$ loses one vibrational quantum the energy is distributed only among the $R_{1}$ and $R_{2}$ stretching modes, which are the dissociative modes. This enhances the efficiency and the probability of the process $\mathrm{Cl}_{2}\left(v^{\prime}\right)-\mathrm{Ne}_{2} \rightarrow \mathrm{Cl}_{2}\left(v^{\prime}-1\right)+2 \mathrm{Ne}$. When the bending modes are included they are expected to accept part of the energy of the vibrational quantum, leaving less energy available for dissociation of the $R_{1}$ and $R_{2}$ stretching modes. As a 
TABLE IV. Calculated and experimental $\mathrm{Cl}_{2}$ vibrational state distributions (in percentage) for the dissociation channels $v^{\prime}-1, v^{\prime}-2$, and $v^{\prime}-3$. Some small percentages of population found in Ref. 15 for the channels $v^{\prime}-4$ and $v^{\prime}-5$, are not listed.

\begin{tabular}{crccc}
\hline \hline & $v^{\prime}$ & $v^{\prime}-1$ & $v^{\prime}-2$ & $v^{\prime}-3$ \\
\hline This & 13 & 0.5 & 93.6 & 5.9 \\
work & 11 & 13.4 & 83.4 & 3.2 \\
& 9 & 44.9 & 53.9 & 1.2 \\
& 8 & 59.6 & 39.7 & 0.6 \\
& 7 & 73.4 & 26.3 & 0.3 \\
& 13 & 1 & 94 & 5 \\
RDQ $^{\mathrm{a}}$ & 12 & 1 & 95 & 5 \\
& 11 & 1 & 96 & 4 \\
& 10 & 1 & 96 & 3 \\
& 9 & 2 & 96 & 2 \\
& 8 & 2 & 96 & 2 \\
Hybrid & 7 & 3 & 96 & 1 \\
$\mathrm{q} / \mathrm{c}^{\mathrm{b}}$ & 13 & 1.3 & 73.7 & 18.4 \\
& 12 & 3.1 & 76.0 & 15.6 \\
& 11 & 7.9 & 75.4 & 13.8 \\
& 10 & 11.8 & 75.1 & 11.1 \\
& 9 & 17.1 & 74.6 & 7.0 \\
Experiment & & & & \\
& 8 & 23.9 & 69.1 & 6.2 \\
& 7 & 25.6 & 68.5 & 5.4 \\
& 9 & 19 & 81 & \\
\hline \hline
\end{tabular}

${ }^{\mathrm{a}}$ Reference 22.

${ }^{\mathrm{b}}$ Reference 15 .

${ }^{\mathrm{c}}$ Reference 5.

result a second or even a third vibrational quantum of energy will be required for complete fragmentation of the two vdW bonds, which increases the probability of the $v^{\prime}-2$ and $v^{\prime}$ -3 channels and decreases the population of $v^{\prime}-1$. This effect leading to an overestimate of the $v^{\prime}-1$ population should also be present in the results of Ref. 22. However, the small $v^{\prime}-1$ populations of Le Quéré and Gray do not seem to show such a behavior, and we shall discuss below the reason of it.

The other factor causing overestimate of the $v^{\prime}-1$ channel is related to the underestimate of the $v^{\prime}-2$ and $v^{\prime}-3$ populations as a result of not considering in our calculations the $\mathrm{Cl}_{2}-\mathrm{Ne}+\mathrm{Ne}$ wave packet components absorbed. We think, however, that this factor has a rather small effect, which is supported by our calculated populations for $v^{\prime}$ $=13$. In fact we obtain $v^{\prime}-1$ and $v^{\prime}-3$ populations for $v^{\prime}=13$ which are lower and higher, respectively, than those of Le Quéré and Gray calculated taking into account the absorbed wave packet components.

It should be noted that two calculations using different methodologies (the present one and that of Bastida et al.) predict a gradual increase of the $v^{\prime}-1$ population as $v^{\prime}$ decreases. This trend is in disagreement with the experimental results of no population in $v^{\prime}-1$ for $v^{\prime} \geqslant 10$, and then a sudden increase of the $v^{\prime}-1$ population for $v^{\prime}<10$. The experimental results seem to indicate that the $v^{\prime}-1$ channel is closed for $v^{\prime} \geqslant 10$. Actually this is consistent with the resonance energy determined experimentally, between -145.6 and $-148.6 \mathrm{~cm}^{-1}$. The discrepancy can be due, again, to possible deficiencies in the potential surface. With the present surface the calculations predict that the $v^{\prime}-1$ channel is energetically closed only for $v^{\prime}=13$, being open for $v^{\prime}<13$ (see $E_{v^{\prime}}$ and $E_{\text {res }}$ energies in Table II). This result is consistent with the gradual increase of the $v^{\prime}-1$ population found here and in Ref. 15 for $v^{\prime}<13$. On the other hand, most of the interaction potentials $(\mathrm{Cl}-\mathrm{Cl}$ and $\mathrm{Cl}-\mathrm{Ne})$ have been tested in full-dimensional quantum calculations of the $\mathrm{Cl}_{2}-\mathrm{Ne}$ predissociation dynamics, and gave good agreement with experiment. ${ }^{16,17,19}$ This remains as an open question.

In the following we shall analyze the reasons of the discrepancy between the present results and those of Ref. 22 for the $v^{\prime}-1$ and $v^{\prime}-2$ populations of $v^{\prime}<13$. In the calculations of Le Quéré and Gray the $v^{\prime}-1$ channel is closed for $v^{\prime}>10$, while in our calculations and in those of Ref. 15 this channel is closed only for $v^{\prime}=13$. The vibrational state $v^{\prime}$ $=10$ is the first one for which the channel $v^{\prime}-1$ is open, and only by $1.14 \mathrm{~cm}^{-1}$ (see Table IV of Ref. 22). The reason is the different resonance energies used in Ref. 22 $\left(\approx 151 \mathrm{~cm}^{-1}\right)$ as compared with those used here and in Ref. $15\left(\approx 125 \mathrm{~cm}^{-1}\right)$. Thus, the small populations found in Ref. 22 for the channel $v^{\prime}-1(1 \%)$ when $v^{\prime}>10$ are due to the fact that this channel is closed. Therefore, here we find a manifestation of the effect of carrying out (or not) the dynamical calculation at the full-dimensional energy of the system, by including the zero-point energy of the modes neglected in the RDQ model.

The result of a small but nonzero population in a channel which is closed $\left(1 \%\right.$ in $v^{\prime}-1$ for $v^{\prime}>10$ in Ref. 22, and $0,5 \%$, and $1.3 \%$ in $v^{\prime}-1$ for $v^{\prime}=13$ in the present calculations and those of Ref. 15, respectively) may appear surprising. It is caused by a small energetic dispersion (of a few $\mathrm{cm}^{-1}$ ) of the initial state, composed of the resonance state of interest and some other states (for which the channel is open), as discussed above. The fact that the population in the closed channel is practically zero indicates that the contamination of these additional states in the initial state is very small.

Despite that the $v^{\prime}-1$ channel is already open for $v^{\prime}$ $\leqslant 10$ in the calculations of Ref. 22 , the populations of this channel are still small (although somewhat larger than for $\left.v^{\prime}>10\right)$. For $v^{\prime} \leqslant 10$ the energy contained in the first vibrational quantum, $E_{v^{\prime}}-E_{v^{\prime}-1}$, is very close to the reduceddimensionality resonance energies of Le Quéré and Gray. Therefore, for an effective dissociation of the two vdW bonds with only one vibrational quantum, each bond should get nearly half of the energy. These events are rather unlikely, being more likely that one vdW stretching mode gets more (or less) energy than $\left(E_{v^{\prime}}-E_{v^{\prime}-1}\right) / 2$, while the other mode gets the remaining energy. Unless that the excess energy in one of the vdW modes is transferred to the other one, fragmentation of the two vdW bonds will require the energy of a second vibrational quantum. The model Hamiltonian of Le Quéré and Gray [Eq. (7)] involves little coupling between the $R_{1}$ and $R_{2}$ modes, since most of the kinetic coupling terms between them have been removed. This makes unlikely energy transfer between the two vdW modes. As a result, dissociation to $\mathrm{Cl}_{2}+2 \mathrm{Ne}$ occurs practically only through the $v^{\prime}-2$ channel, even for the $v^{\prime}$ states where the $v^{\prime}-1$ channel is open. This explains the small population 
found in Ref. 22 in the $v^{\prime}-1$ channel also for $v^{\prime} \leqslant 10$. In fact the authors of Ref. 22 carried out test calculations increasing the coupling between the $R_{1}$ and $R_{2}$ modes by decreasing the angle between the two vdW bonds. They found a substantial increase in the $v^{\prime}-1$ population, which supports the above discussion.

Therefore, the $v^{\prime}-1$ populations calculated by Le Quéré and Gray seem to be underestimated, either because the $v^{\prime}$ -1 channel is artificially closed due to neglecting the bending zero-point energy, or because the coupling between the $R_{1}$ and $R_{2}$ modes is underestimated. The present RDQ model, in addition to include the bending zero-point energy, which prevents to close artificially the $v^{\prime}-1$ channel, also considers all the coupling terms between the modes, as in the full Hamiltonian. Moreover, the average of these couplings over the angular function $\mathcal{F}_{v^{\prime}}$ of Eq. (15) takes into account the weight of all the angular configurations, not only the equilibrium one. This permits a more flexible description of the interaction between the vdW modes. As a consequence, the model proposed here yields higher $v^{\prime}-1$ populations, and it is able to reproduce the experimental result that for $v^{\prime}=7$ dissociation of $\mathrm{Cl}_{2}-\mathrm{Ne}_{2}$ occurs predominantly through the $v^{\prime}-1$ channel. We note that not even the fulldimensional hybrid quantum-classical calculations, ${ }^{15}$ which in general provide good agreement with the experimental data, describes this result.

In Fig. 4 wave packet probability density plots are shown for the channels $v^{\prime}-1$ and $v^{\prime}-2$, for $v^{\prime}=7$, at $t$ $=4$ ps. These plots are comparable to those shown in Fig. 4 of Ref. 22. Appreciable density is found in the $v^{\prime}-1$ channel around the line $R_{1}=R_{2}$. Such a density is consistent with direct dissociation into $\mathrm{Cl}_{2}\left(v^{\prime}-1\right)+2 \mathrm{Ne}$ by excitation of the vdW symmetric stretching mode. With the same scale and number of contours the wave packet in channel $v^{\prime}-2$ [Fig. 4(b)] does not show density around $R_{1}=R_{2}$. It means that production of $\mathrm{Cl}_{2}$ by sequential dissociation of $\mathrm{Cl}_{2}-\mathrm{Ne}_{2}$ through $v^{\prime}-2$ is less intense than direct dissociation through $v^{\prime}-1$, consistently with the vibrational populations of Table IV. This is the opposite behavior to that found in the wave packet density plots of Fig. 4 of Ref. 22, as expected.

\section{CONCLUSIONS}

A reduced-dimensionality quantum model is proposed to simulate the vibrational predissociation of $\mathrm{BC}-\mathrm{Rg}_{2} \mathrm{vdW}$ complexes. The main features of the model are (a) the zeropoint energy of the modes neglected in the model is incorporated in a systematic way by averaging the full Hamiltonian over the initial state dependence on these modes; (b) the reduced Hamiltonian obtained in this way conserves all the terms of the full Hamiltonian, therefore, retaining all the coupling terms between the modes explicitly considered in the model; (c) by averaging over the dependence on the neglected modes the reduced Hamiltonian takes into account the weight of all the configurations in those modes, and not only the equilibrium one, as typically done in other reduceddimensionality models. The model has been applied to simulate the predissociation dynamics of $\mathrm{Cl}_{2}-\mathrm{Ne}_{2}$, by considering the stretching modes of the complex, while the bending modes are neglected. The results are compared to available

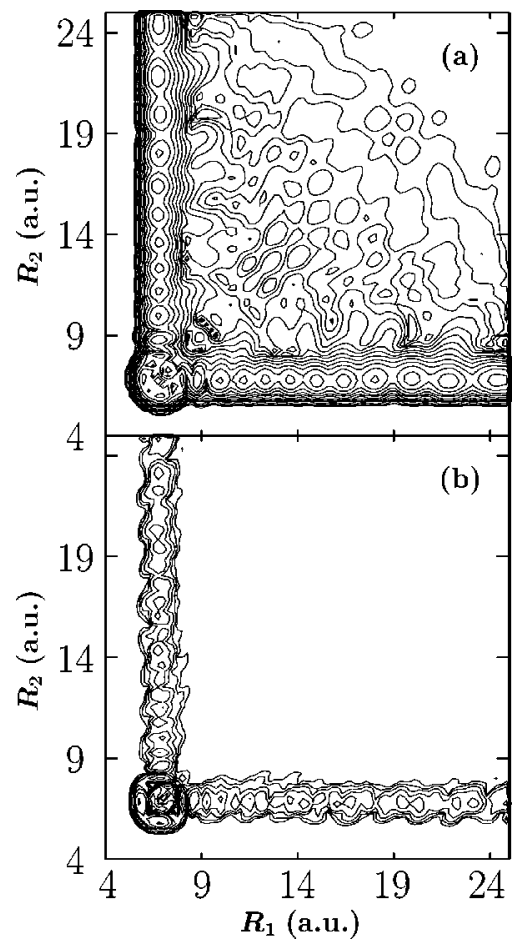

FIG. 4. Wave packet probability densities at $t=4 \mathrm{ps}$ for the $\mathrm{Ne}_{2} \mathrm{Cl}_{2}\left(v^{\prime}\right.$ $=7$ ) propagation. The dissociation channels $v^{\prime}-1$ (a) and $v^{\prime}-2$ (b) are shown. In the figure 14 contour levels are displayed where the highest one corresponds to the maximum density in the $v^{\prime}-1$ channel. Each successive contour is $(1 / 2)$ the previous one. With this scale of contours the density in the $v^{\prime}-3$ channel cannot be displayed.

experimental data, and to previous calculations using a reduced-dimensionality quantum model and a fulldimensional quantum-classical approach.

The quantities investigated are the resonance decay lifetimes of the complex and the vibrational distributions of the $\mathrm{Cl}_{2}$ product fragment. These magnitudes are calculated for five $\mathrm{Cl}_{2}-\mathrm{Ne}_{2}$ resonances corresponding to the initial vibrational excitation of $\mathrm{Cl}_{2} v^{\prime}=7,8,9,11,13$. The calculated lifetimes are found to be an upper limit as compared with the experimental and previously calculated ones. This is consistent with the reduced dimensionality of the model and with the inclusion of the zero-point energy of the neglected modes. The behavior of an increasing lifetime with decreasing $v^{\prime}$ excitation is reproduced by the model. However, a more quantitative, reasonable agreement with experiment is only found for $v^{\prime}=11,13$, and deteriorates gradually for lower $v^{\prime}$, as a consequence of an increasingly important role played by the neglected bending modes. The model predicts correctly the experimental trend for the $\mathrm{Cl}_{2}$ vibrational distributions, namely that for the higher $v^{\prime}$ states the $v^{\prime}-2$ dissociation channel is the dominant one, but as $v^{\prime}$ decreases the situation gradually inverts and $v^{\prime}-1$ becomes dominant. None of the two previous calculations predicted the dominance of the $v^{\prime}-1$ channel for lower $v^{\prime}$ excitations.

Although the present work reproduces the main features of the dynamics, it evidences the importance of considering the neglected angular modes, mainly as $v^{\prime}$ decreases. The effect of such modes can be incorporated to the present model at an affordable computational price, albeit only par- 
tially, within the time-dependent self-consistent-field scheme. ${ }^{32}$ Work on this line is currently in progress. In addition, the potential surface used for $\mathrm{Cl}_{2}-\mathrm{Ne}_{2}$ might need some refinement in order to fit the experimental results.

Finally, the model proposed is not restricted to the vibrational predissociation of vdW complexes, and can be applied to other dynamical processes, as well as to systems larger than tetraatomic ones. The only requirement is the calculation of the full-dimensional initial state, needed to obtain the reduced Hamiltonian. The presently available quantum diffusion Monte Carlo techniques make possible the calculation of the full-dimensional initial states for larger systems. Therefore, we envision a wide applicability of the present method.

\section{ACKNOWLEDGMENTS}

Professor P. Villarreal is acknowledged for making available the code to calculate the initial resonance states of $\mathrm{Cl}_{2}-\mathrm{Ne}_{2}$. This work was supported by D.G.I.C.Y.T., Spain, Grant No. PB95-0071. One of the authors (M.C.) acknowledges financial support for a stay of six months in Madrid to the European network Training and Mobility of Researchers (TMR), Grant No. HPRN-CT-1999-00005.

${ }^{1}$ W. Sharfin, K. E. Johnson, L. Warton, and D. H. Levy, J. Chem. Phys. 71, 1292 (1979).

${ }^{2}$ J. E. Kenny, K. E. Johnson, W. Sharfin, and D. H. Levy, J. Chem. Phys. 72, 1109 (1980).

${ }^{3}$ B. A. Swartz, D. E. Brinza, C. M. Western, and K. C. Janda, J. Phys. Chem. 88, 6272 (1984).

${ }^{4}$ J. C. Drobits and M. I. Lester, J. Chem. Phys. 86, 1662 (1987).

${ }^{5}$ S. R. Hair, J. I. Cline, C. R. Bieler, and K. C. Janda, J. Chem. Phys. 90, 2935 (1989).

${ }^{6}$ D. D. Evard, C. R. Bieler, J. I. Cline, N. Sivakumar, and K. C. Janda, J. Chem. Phys. 89, 2829 (1988); C. R. Bieler, D. D. Evard, and K. C. Janda, J. Phys. Chem. 94, 7452 (1990).

${ }^{7}$ W. D. Sands, C. R. Bieler, and K. C. Janda, J. Chem. Phys. 95, 729 (1991).

${ }^{8}$ D. M. Willberg, M. Gutmann, J. J. Breen, and A. H. Zewail, J. Chem.
Phys. 96, 198 (1992); M. Gutmann, D. M. Willberg, and A. H. Zewail, ibid., 97, 8037 (1992); 97, 8048 (1992).

${ }^{9}$ A. García-Vela, P. Villarreal, and G. Delgado-Barrio, J. Chem. Phys. 92, 6504 (1990)

${ }^{10}$ A. García-Vela, P. Villarreal, and G. Delgado-Barrio, J. Chem. Phys. 94, 7868 (1991)

${ }^{11}$ A. García-Vela, J. Rubayo-Soneira, G. Delgado-Barrio, and P. Villarreal, J. Chem. Phys. 104, 8405 (1996).

${ }^{12}$ Z. Li, A. Borrmann, and C. C. Martens, J. Chem. Phys. 97, 7234 (1992).

${ }^{13}$ M. I. Hernández, A. García-Vela, C. García-Rizo, N. Halberstadt, P. Villarreal, and G. Delgado-Barrio, J. Chem. Phys. 108, 1989 (1998).

${ }^{14}$ S. Fernández Alberti, N. Halberstadt, J. A. Beswick, A. Bastida, J. Zúñiga, and A. Requena, J. Chem. Phys. 111, 239 (1999).

${ }^{15}$ A. Bastida, B. Miguel, J. Zúñiga, A. Requena, N. Halberstadt, and K. C. Janda, J. Chem. Phys. 111, 4577 (1999).

${ }^{16}$ N. Halberstadt, J. A. Beswick, and K. C. Janda, J. Chem. Phys. 87, 3966 (1987)

${ }^{17}$ J. I. Cline, N. Sivakumar, D. D. Evard, C. R. Bieler, B. P. Reid, N. Halberstadt, S. R. Hair, and K. C. Janda, J. Chem. Phys. 90, 2605 (1989).

${ }^{18}$ O. Roncero, J. A. Beswick, N. Halberstadt, P. Villarreal, and G. DelgadoBarrio, J. Chem. Phys. 92, 3348 (1990).

${ }^{19}$ S. K. Gray and C. E. Wozny, J. Chem. Phys. 94, 2817 (1990).

${ }^{20}$ A. Rohrbacher, T. Ruchti, K. C. Janda, A. A. Buchachenko, M. I. Hernández, T. González-Lezana, P. Villarreal, and G. Delgado-Barrio, J. Chem. Phys. 110, 256 (1999).

${ }^{21}$ P. Villarreal, A. Varadé, and G. Delgado-Barrio, J. Chem. Phys. 90, 2684 (1989).

${ }^{22}$ F. Le Quéré and S. K. Gray, J. Chem. Phys. 98, 5396 (1993).

${ }^{23}$ M. I. Hernández, N. Halberstadt, W. D. Sands, and K. C. Janda, J. Chem. Phys. 113, 7252 (2000).

${ }^{24}$ O. Roncero, G. Delgado-Barrio, M. I. Hernández, J. Campos-Martínez, and P. Villarreal, Chem. Phys. Lett. 246, 187 (1995).

${ }^{25}$ J. Campos-Martínez, M. I. Hernández, O. Roncero, P. Villarreal, and G. Delgado-Barrio, Chem. Phys. Lett. 246, 197 (1995).

${ }^{26}$ P. Villarreal, O. Roncero, and G. Delgado-Barrio, J. Chem. Phys. 101, 2217 (1994).

${ }^{27}$ R. N. Zare, Angular Momentum (Wiley, New York, 1988).

${ }^{28}$ A. R. Edmonds, Angular Momentum in Quantum Mechanics (Princeton University Press, Princeton, 1957).

${ }^{29}$ H. Tal-Ezer and R. Kosloff, J. Chem. Phys. 81, 3967 (1984).

${ }^{30}$ V. Engel, Chem. Phys. Lett. 189, 76 (1992).

${ }^{31}$ P. Pernot and W. A. Lester, Int. J. Quantum Chem. 40, 577 (1991).

${ }^{32}$ A. García-Vela, Chem. Phys. Lett. 290, 155 (1998); J. Chem. Phys. 111, 8286 (1999). 